\title{
Searches for Supersymmetry and Other Exotica at the LHC
}

\author{
D. Milstead ${ }^{\mathrm{a}}$ on behalf of the ATLAS and CMS collaborations \\ ${ }^{\text {a}}$ Fysikum, Stockholms Universitet, Stockholm, Sweden.
}

The Large Hadron Collider (LHC) has opened up a new kinematic regime. Using high energy proton-proton collisions it is possible to search for evidence of hitherto unobserved particles and processes at the TeV scale. This paper describes search strategies for a range of exotic phenomena proposed within supersymmetry and other theories.

\section{Introduction}

Searches for physics beyond the Standard Model are made whenever a new energy regime becomes available. The Large Hadron Collider (LHC) allows searches for phenomena associated with multi-TeV energy scales. Predictions of hitherto unobserved particles and processes in this energy regime are motivated by a number of arguments. For example, a weakly interacting massive particle (WIMP) could serve as a candidate for dark matter. Furthermore, the Standard Model suffers from an arguably unnatural fine tuning of the renormalised Higgs mass. Theories which address this problem, such as supersymmetry (SUSY) [1], typically predict that new physics processes would be manifest at the LHC.

Although there exist robust arguments to anticipate some type of new phenomena at the LHC, it is impossible to quantitatively prescribe the form they will take. The general purpose detectors at the LHC, ATLAS and CMS, need therefore to be prepared to search for a range of different possible signatures. For example, an exotic heavy stable particle could manifest itself as an indirectly observed WIMP or as a so-called Stable ${ }^{1}$ Massive Particle [2] (SMP), which is observed via its interactions in the detector. The presence of decayed exotic particles could be inferred via a resonant linewidth or via specific event topologies associated with certain decay products. Although it is important that searches are as generic as pos-

${ }^{1}$ The term stable is taken to mean that the particle will not decay during its traversal of a detector. sible, models which prescribe specific signatures are helpful in providing a theoretical framework in which results can be interpreted. In this paper a selected overview of search strategies for different types of signatures is given. It is also shown how the results can be interpreted in models of SUSY, extra dimensions [3-5] and string balls [6].

\section{R-parity Conserving Supersymmetry}

As mentioned earlier, TeV-scale SUSY is theoretically favoured by many since it stabilises the Higgs mass. Furthermore, in the case of $R$-parity conserving supersymmetry, a dark matter candidate, a so-called WIMP is available, which would be observed as excess missing transverse energy in a detector. Since the most minimal supersymmetric extensions to the Standard Model contain over one hundred free parameters, a number of different classes of signatures associated with the production of so-called sparticles can be anticipated. A comprehensive approach therefore requires that various event topologies are studied. Such work is being conducted by both ATLAS [7] and CMS [8].

Discovery sensitivities are presented here for several different topologies and are taken to be representative of the larger body of work which covers a more complete range of final states. The channel containing at least one lepton, three jets and missing transverse energy greater than 80 $\mathrm{GeV}$ has been studied by ATLAS. Figure 1 shows the results in the context of a mSUGRA model in which squarks and gluinos are assumed to be 
mass-degenerate. The current limit on squark and gluino masses from the Tevatron within a mSUGRA approach in which these particles are assumed to have the same mass is $\sim 380 \mathrm{GeV}[9]$. The integrated luminosity required for a discovery (i.e. at $5 \sigma$ significance) is shown as a function of centre-of-mass energy and it is seen that discovery is possible with luminosity of order 100 $\mathrm{pb}^{-1}$ at $7 \mathrm{TeV}$ centre-of-mass energy.



Figure 1. The integrated luminosity required for a discovery at $5 \sigma$ significance of SUSY according to a mSUGRA model, shown as a function of centre-of-mass energy. The discovery potential corresponds to a channel leading to one lepton, at least three jets and missing transverse energy greater than $80 \mathrm{GeV}$.

The discovery potential in the so-called allhadronic channel is shown in Figure 2. A selection was imposed in which events containing high momentum electrons and muons were excluded and a minimum of three jets was required. Further requirements were made on missing transverse energy and on the topology of the events [8]. The projected regions in mSUGRA parameter space $m_{0}$ and $m_{1 / 2}$ [1] which can be excluded at $95 \%$ confidence level (CL) are shown for different assumed values of integrated luminosity.



Figure 2. Estimated 95\% CL exclusion limits in mSUGRA parameter space for different assumed values of integrated luminosity. The plot corresponds to the all-hadronic channel.

\section{Resonance Production}

Exotic resonances have been postulated in a number of models, such as $R$-parity violating SUSY [10] and theories of extra dimensions [3-5]. In Figure 3 the expected sensitivity at ATLAS to the production and muonic decay of a sequential Standard Model-like Z' [11] is shown for masses of 1 and $1.5 \mathrm{TeV}$, as a function of centre-of-mass energy. The total integrated luminosity which is required to exclude such particles at a $95 \%$ confidence level, and the luminosity needed to observe ten of them, which typically offers a greater significance than $5 \sigma$, are shown. For comparison, the current Tevatron lower limit is around $1 \mathrm{TeV}$.

Figure 4 shows the CMS estimates of the integrated luminosity required for a $5 \sigma$ significance observation of different $Z^{\prime}$ bosons and Randall-Sundrum gravitons [4]. Sequential Standard Model-like Z' bosons (SSM Z') are considered, as are bosons predicted within an E6 GUT model [11] $\left(\mathrm{Z}_{\psi}^{\prime}\right)$ and Randall-Sundrum (RS) gravitons corresponding to different coupling values. For an integrated luminosity of around 




Figure 3. Required integrated luminosity to observe ten $Z^{\prime}$ events or exclude $Z^{\prime}$ production at a $95 \%$ CL. Plots are shown for different assumed $\mathrm{Z}^{\prime}$-masses and as a function of centre-of-mass energy.

$100 \mathrm{pb}^{-1} \mathrm{CMS}$ has a sensitivity beyond the Tevatron limits.

\section{String Balls}

It has been proposed that mini-black holes can be produced at the LHC [12]. Such objects can be treated according to general relativity (GR) prescriptions for a black hole mass, $M_{B H}$, greater than around $5 M_{D}$, where $M_{D}$ is the fundamental Planck scale in higher dimensions. Below the GR threshold, quantum gravity effects are expected to manifest. It has been shown that highly excited string states, known as string balls [6], produced below the GR threshold, could have a cross section comparable to that of a black hole. Furthermore, black holes produced at the LHC could evolve into these string states.

High-mass string ball states can be detected with selections on specific event topologies. In



Figure 4. Required integrated luminosity for an observation at $5 \sigma$ significance of SSM Z $\mathrm{Z}^{\prime}$ bosons, $\mathrm{Z}_{\psi}^{\prime}$ bosons, and RS gravitons corresponding to different coupling values. The luminosity is shown as a function of the particle mass.

Figure 5 the event yields for a string ball model [13] and background processes are shown as a function of the invariant mass within an event. The invariant mass was formed from reconstructed muons, electrons, photons and jets [14]. The sum of missing transverse energy and the scalar sum of transverse momentum of final state objects was required to be greater than $2.4 \mathrm{TeV}$, and a electron or muon with a high transverse momentum $\left(p_{T}>100 \mathrm{GeV}\right)$ was required. As can be seen little background remains. Although this study was performed assuming a centre-of-mass energy of $10 \mathrm{TeV}$, the conclusions that such striking objects can be seen and background strongly suppressed also hold for a $7 \mathrm{TeV}$ centre-of-mass energy.

\section{Stable Massive Particles}

The discovery of SMPs which interact in a detector would be of fundamental significance. Such particles are predicted in a range of SUSY mod- 


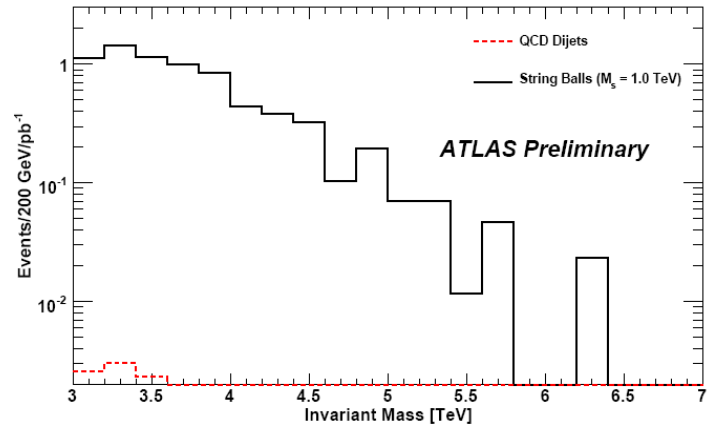

Figure 5. The invariant mass of selected final state objects for string ball and background events.

els, such as Split-supersymmetry and GMSB [2]. Since such particles are expected to be slow $(\beta \ll$ $1)$, searches can be made based on direct timeof-flight measurements and anomalous continuous energy loss. Furthermore, in the case of SMPs formed from a coloured object, so-called $R$-hadrons, hadronic scattering [15-17] can lead to striking event topologies in which the SMP changes electric charge during its propagation through the detector.

Using a tracker-based analysis, CMS evaluated the integrated luminosity required to exclude, at 95\% confidence level, gluino and stop-based $R$ hadrons of various masses [8]. These results are shown in Figure 6. For comparatively modest amounts of integrated luminosity of order $\mathrm{pb}^{-1}$ it is possible to exceed the direct limits set by the Tevatron experiments [2]. Similar conclusions were drawn by the ATLAS experiment [18].

The charge-exchange properties of $R$-hadrons was also studied by ATLAS. In Figure 7, the ratio of transverse momenta (signed with the reconstructed electric charge of the particle) in the inner and muon tracking systems is shown. As can be seen, the gluino-based $R$-hadrons can reverse the sign of their charge This property would be used in search strategies [18].

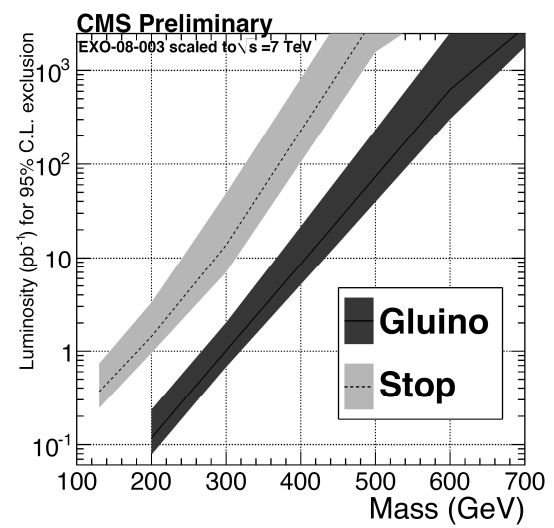

Figure 6. Required integrated luminosity to exclude gluino and stop-based $R$-hadrons as a function of the particle mass.

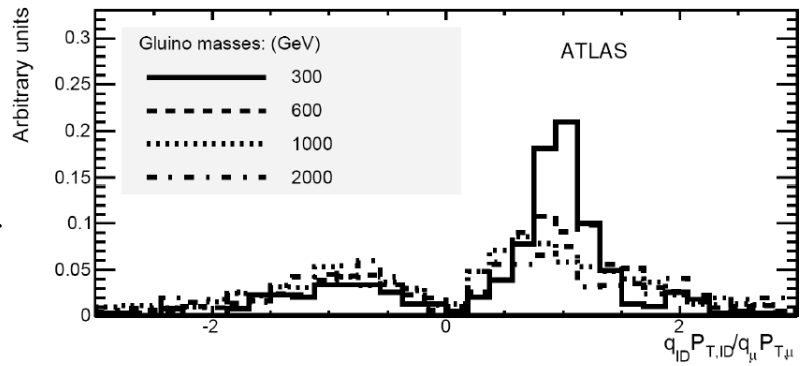

Figure 7. The ratio of signed transverse momenta in the inner and muon tracking systems for gluino-based $R$-hadrons.

\section{Stopped Gluinos}

In most models of SMPs, the particle is metastable, possessing a sufficiently long lifetime so as to traverse a detector and decay at a later stage. While most SMPs would decay outside of the detector, a small fraction of SMPs, typically those produced on or around their kinematic production threshold, would be stopped owing to energy loss via electromagnetic interactions and (if the SMPs are hadronic) reactions with nuclei in the detectors. Most would stop in the dense calorimeter material.

CMS has studied the possibility of looking for 
decays of stopped gluinos [19]. The search would be made during gaps between beam crossings and the inter-fill period between beam-dump and reinjection. A jet-based trigger looking for energy depositions characteristic of gluino decays would be used. In Figure 8 the expected signal significance is shown for gluinos of mass $300 \mathrm{GeV}$ for various lifetimes. Results are shown for instantaneous luminosities of $10^{32} \mathrm{~cm}^{-2} \mathrm{~s}^{-1}$ and $10^{31} \mathrm{~cm}^{-2} \mathrm{~s}^{-1}$. Discovery beyond Tevatron limits [20] is possible with a couple of weeks of running at the higher luminosity.
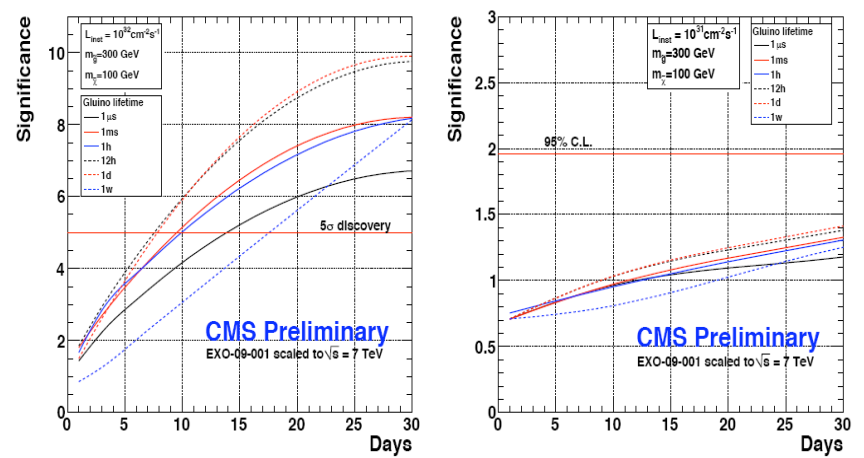

Figure 8. The discovery potential for a long-lived gluino of mass $300 \mathrm{GeV}$ which stops in the CMS calorimeter in a $7 \mathrm{TeV}$ run at an instantaneous luminosity of $10^{32} \mathrm{~cm}^{-2} \mathrm{~s}^{-1}$ (left) and $10^{31} \mathrm{~cm}^{-2} \mathrm{~s}^{-1}$ (right).

\section{Summary}

The LHC has opened up a new kinematic region. ATLAS and CMS have developed search strategies to look for exotic phenomena associated with a range of different event signatures. It was shown that sensitivity to hitherto unobserved particles and processes expected in various models of physics beyond the Standard Model is expected during the early running period of the LHC.

\section{REFERENCES}

1. S. P. Martin, arXiv:hep-ph/9709356.

2. M. Fairbairn, A. C. Kraan, D. A. Milstead, T. Sjostrand, P. Z. Skands and T. Sloan, Phys. Rept. 438, 1 (2007) [arXiv:hep$\mathrm{ph} / 0611040]$.

3. A. Leike, Phys. Rept. 317 (1999) 143 [arXiv:hep-ph/9805494].

4. L. Randall and R. Sundrum, Phys. Rev. Lett. 83, 3370 (1999) [arXiv:hep-ph/9905221].

5. L. Randall and R. Sundrum, Phys. Rev. Lett. 83, 4690 (1999) [arXiv:hep-th/9906064].

6. S. Dimopoulos and R. Emparan, Phys. Lett. B 526 (2002) 393 [arXiv:hep-ph/0108060].

7. [The ATLAS Collaboration], ATL-PHYSPUB-2009-084.

8. [The CMS Collaboration], CMS-NOTE-2010008.

9. T. Aaltonen et al. [CDF Collaboration], Phys. Rev. Lett. $102 \quad$ (2009) 121801 [arXiv:0811.2512 [hep-ex]].

10. R. Barbier et al., Phys. Rept. 420 (2005) 1 [arXiv:hep-ph/0406039].

11. M. Cvetic and S. Godfrey, arXiv:hepph/9504216.

12. S. Dimopoulos and G. L. Landsberg, Phys. Rev. Lett. 87 (2001) 161602 [arXiv:hepph/0106295].

13. D. M. Gingrich and K. Martell, Phys. Rev. D 78 (2008) 115009 [arXiv:0808.2512 [hep-ph]].

14. [The ATLAS Collaboration], ATL-PHYSPUB-2009-011.

15. A. C. Kraan, Eur. Phys. J. C 37 (2004) 91 [arXiv:hep-ex/0404001].

16. Y. R. de Boer, A. B. Kaidalov, D. A. Milstead and O. I. Piskounova, J. Phys. G 35, 075009 (2008) [arXiv:0710.3930 [hep-ph]].

17. R. Mackeprang and D. Milstead, Eur. Phys. J. C 66, 493 (2010) [arXiv:0908.1868 [hep-ph]].

18. [The ATLAS Collaboration], arXiv:0901.0512 [hep-ex].

19. [The CMS Collaboration], Note EXO-09-001.

20. V. M. Abazov et al. [D0 Collaboration], Phys. Rev. Lett. 99 (2007) 131801 [arXiv:0705.0306 [hep-ex]]. 